DOI 10.32370/2018_07_6

\title{
Another experiment rejects Ampere's Law and supports the STOE model
}

\author{
J.C. Hodge ${ }^{1 *}$ \\ ${ }^{1}$ Retired, 477 Mincey Rd., Franklin, NC, 28734
}

\begin{abstract}
The Scalar Theory of Everything (STOE) posits the components of the universe emerge to cause gravity and electromagnetic effects. An experiment to test Ampere's law and the STOE was performed. The data shows the STOE model is very close to the actual measurements. The traditional magnetostatics Biot-Savart Law is rejected. The STOE model of magnetostatics is not rejected and is supported.
\end{abstract}

keywords: STOE, Theory of Everything, magnetostatics, Biot-Savart Law

\section{Introduction}

Maxwell's Equations classically describe the relationships of magnetic (B) and electric (E) fields. They are inexact and seem to not apply to situations involving individual photons.

The Scalar Theory of Everything (STOE) suggests the hods have a greater plenum density $\rho$ held at a maximum on one side and a minimum on the other side which is the magnetic effect. The magnetic field of classical physics is a cloud of hods with the same orientation. A photon is a column of hods. The density of hods is the $B$ intensity. A current of electrons in a wire ejects hods perpendicular to the wire and the current direction. An experiment rejected the Biot-Savart (Ampere's) Law and did not reject the STOE model (3). The Biot-Savart Law for a magnetic field $\vec{B}_{b}(\vec{R})$ at a position $\vec{R}$ from a element $\mathrm{d} L$ of the wire caused by a current $\vec{I}$ in the wire is

$$
\vec{B}_{b}(\vec{r})=\frac{\mu_{0}}{4 \pi} \int_{c} \frac{\vec{I} \mathrm{~d} L \times \vec{R}}{|\vec{R}|^{3}}
$$

where $\mu_{0}$ is the magnetic constant and the integration is considered to be for an infinitely long wire.

This paper demonstrates the effect of the STOE model of a current in a solenoid. The terms and development of the STOE model may be viewed at Vixrapedia and Hodge (2).

*E-mail: jchodge@frontier.com 


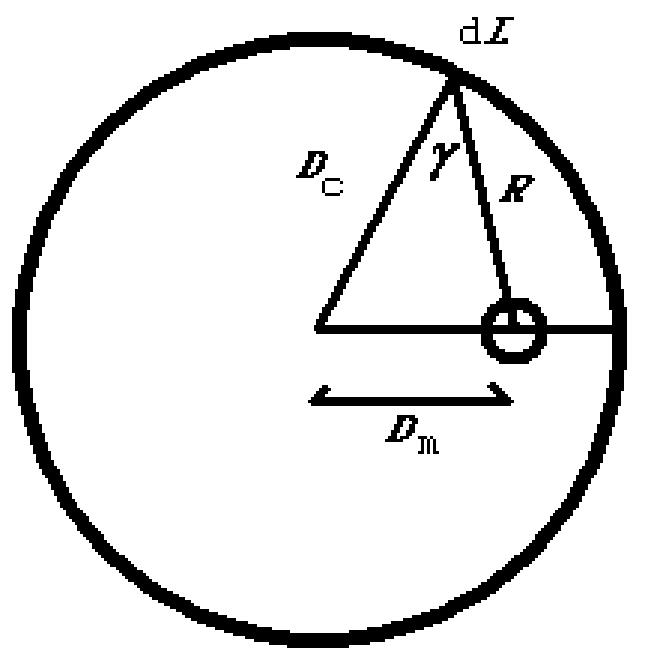

Figure 1: Diagram showing the symbols used when the small, round magnet is in a solenoid.

\section{Model}

The STOE effect of a current in a circular loop of wire is within an angle $\pm \theta$ from the radial direction from each point $\mathrm{d} L$ along the wire. Figure 1 is a diagram of that part of the loop (big circle) which has an effect on the magnet (small circle).

Hods are emitted from moving electrons with a uniform N-S (north - south) pole direction (3). As the hods travel from the emission point, their magnetic fields repel causing the hod distribution to disperse laterally and radially. The hod distribution becomes spherical. Therefore, the distribution density becomes proportional to $1 /$ distance $^{2}$. The hod distribution spreads with an angle $\theta$ from the normal to the $\vec{I} d L$.

The force $W$ on the magnet is proportional to the number of hods (density times volume) in the magnet. The magnetic $\vec{B}_{s}$ in the STOE model for each point in the magnet is

$$
\vec{B}_{s}(\vec{R})=\delta \vec{B}_{b}(\vec{R}),
$$

where

$$
\begin{array}{rlr}
\delta & =1 & \theta>\gamma \\
& =0 & \theta \leq \gamma
\end{array}
$$




\section{Experiment}

(All measurement are $\pm 10 \%$ unless otherwise stated).

A solenoid was formed with 60 turns of 22 AWG wire with a diameter of $16 \mathrm{~cm}\left(D_{\mathrm{c}}=8 \mathrm{~cm}\right)$ and a height of $9.8 \mathrm{~cm}$. Disc magnets ("Nexlevel super strong mini" $6 \mathrm{~mm}$ wide X $2 \mathrm{~mm}$ thick) are assembled into a column $5 \mathrm{~cm}$ long ("bar magnet"). The bar magnet is placed at the bottom plane of the solenoid and on a wood block which is place on a gram-weight (gw) scale (AWS-100, Digital scale) with a tolerance of \pm 0.02 gw. The weight measured $W$ (gw) is the difference of the meter reading without current and the meter reading with current. The absolute value of the readings with current in both directions are averaged which reflects averaging a force that increases $W$ and a force that decreases $W$.

Simplifications that make the experiment easier to analyze are the measured values at a point are compared to the measured value $W_{0}$ at the center of the solenoid, the action is considered to be at the center of the magnet, and the calculation is reduced to the plane at one end of the solenoid. The analysis was with an Excel program.

Figure 2 shows the data of $W / W_{0}$ versus the $D_{\mathrm{m}}$ and the calculated values for the STOE model. The traditional Biot-Savart Law (Ampere's Law) model suggests the entire loop affects the magnet at all positions of the magnet.

\section{Discussion and Conclusion}

The magnetic "field" according to the STOE is the density distribution of hods with the same orientation. This is like the electric "field" being the density of plenum vortices (cones and rings). The hods generate vortices in the plenum as they move. The behavior of hods far from the emitting current in antennas is the study of the electromagnetic field (EM). The description of the EM field from a dipole antenna array is the basis for the STOE model of photon diffraction.

Figure 2 shows the STOE theoretical line with a variation about the data line. This is because the theoretical calculation involved an assumption that the action is at the point in the center of the magnet. If this point is just inside the $\theta$, the whole magnet is counted. The next $\vec{I} d L$ may have the point outside the $\theta$ value and is not be counted.

The data shows the STOE model is very close to the actual measurements. The traditional magnetostatics Biot-Savart Law is rejected. The STOE model of magnetostatics is not rejected and is supported. 


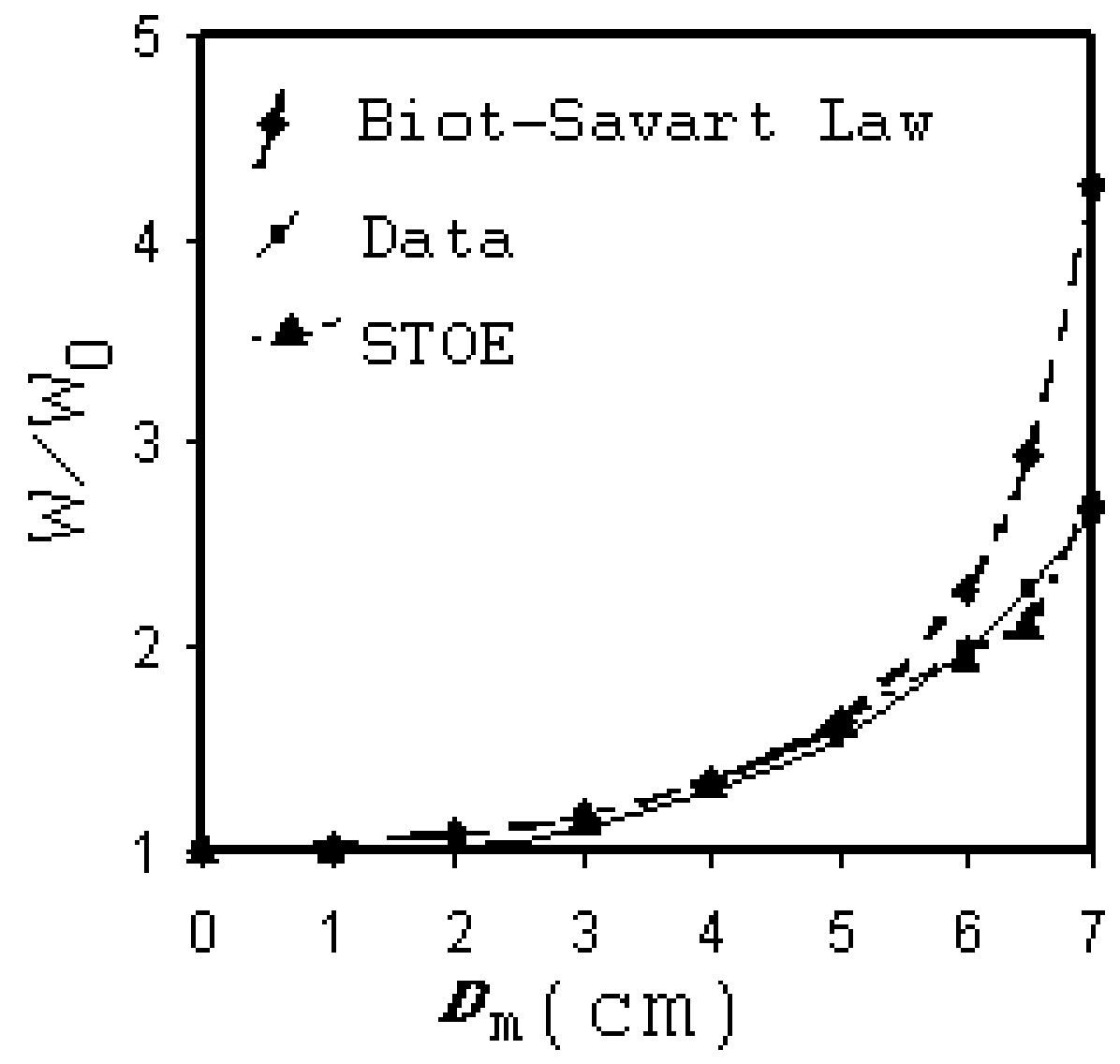

Figure 2: Measured data (solid line) and the STOE calculated data (triangles with dotted line) graph with the Biot-Savart Law calculations added. The $\theta=0.82$ radians. 


\section{REFERENCES}

\section{References}

[1] Hodge, J.C., 2012, Photon diffraction and interference, IntellectualArchive, Vol.1, No. 3, P. 20,. http://intellectualarchive.com/?link=item\&id $=597$

[2] Hodge, J.C., 2015d, Universe according to the STOE, http://intellectualarchive.com/?link=item\&id=1648

[3] Hodge, J.C. Magnetostatics relation to gravity with experiment that rejects Biot-Savart Law. IntellectualArchive, 2018, v.7(3), p. 1-8. http://intellectualarchive.com/?link=item\&id=1945.

[Vixrapedia] Hodge, J.C. Theory of Everything (STOE), http://www.vixrapedia.org/wiki/Theory_of_Everything_(STOE). 